\title{
THE EFFECTS OF A RAPIDLY-FLUCTUATING RANDOM ENVIRONMENT ON SYSTEMS OF INTERACTING SPECIES*
}

\author{
BENJAMIN S. WHITE†
}

\begin{abstract}
Some models of interacting species in a random environment are analyzed. Approximate solutions of the stochastic differential or delay-differential equations describing the systems are obtained, on the assumption that the random environment is fluctuating rapidly.
\end{abstract}

\section{PART I}

1. Introduction. The theory of interacting species in a random environment leads to stochastic differential equations. We will show how to solve these equations approximately when the randomness is rapidly-varying. The solution is approximated by a deterministic path with random fluctuations superimposed on it, and is constructed in two steps: first, equations are obtained for the deterministic path. They are nonlinear, wholly deterministic, and are often just the corresponding equations for the system of species in a fixed environment. Thus the deterministic theory may be utilized in constructing a first approximation for the stochastic solution. Second, linear stochastic equations are obtained for the Gaussian random fluctuations about the deterministic path. The linearity of these equations allows for a greatly simplified treatment of the stochastic component; moreover, because of this simplicity, it is possible to treat some processes with after-effect-systems of species with time-delayed interactions, co-existing in a random environment.

We generalize somewhat the more usual "white noise" assumptions for the environment (see May [7], [8]) by postulating randomness which is rapidlyvarying (but possibly smooth) and forgetful of the past (but not instantaneously). Other than this, only two averaged moments of the environmental process enter into the calculations, and further specification of the environmental statistics will not change the results.

For clarity we will solve some specific equations. However, the results of this method are largely model-independent. Thus many mathematical models which agree in certain well-defined ways can be seen to give equivalent results to this order of approximation.

The calculations that follow are valid for any fixed, finite time interval provided only that the environment is sufficiently rapidly-varying. Since they are not valid for infinite time, this would seem to preclude any statements about long-time "equilibrium" population statistics. However, in many cases when environmental randomness is introduced into a deterministically stable system, we shall be able to compute an approximate "pseudo-equilibrium" probability distribution which will be noticeable within our finite observation time. The existence of this intermediate limit, which may be quite different from a true

* Received by the editors May 4, 1976.

$\dagger$ Courant Institute of Mathematical Sciences, New York University, New York, New York. Now at Department of Applied Mathematics, California Institute of Technology, Pasadena, California 91125. This work was supported by the National Science Foundation under Grant MPS 75-09837. 
infinite time equilibrium, raises the question of which limit is observed when we view an apparently stable biological system in a random environment.

\section{Results for systems without delayed interactions.}

2.1. The averaged and fluctuation equations. Let $x_{i}(t), i=1,2, \cdots, d$, be a continuous approximation to the number of individuals of species $i$ alive at time $t$, and let $\mathbf{X}=\left(x_{1}, \cdots, x_{d}\right)$. We consider a deterministic description of population growth of the form

$$
\frac{d}{d t} \mathbf{X}(t)=\mathbf{F}(t, \mathbf{X}(t) ; \boldsymbol{\gamma})
$$

Here the vector function $\mathbf{F}$ describes the dynamics of the species interactions, and $\boldsymbol{\gamma}=\left(\gamma_{1}, \cdots, \gamma_{r}\right)$ is a vector of parameters which describe the environment. The dependence of the interactions on $t$ as well as on $\mathbf{X}(t)$ allows for the environment to change with time, but in a deterministic way.

In a random environment, the parameters $\boldsymbol{\gamma}$ will fluctuate randomly in time, and we assume these fluctuations are rapid. This is expressed by writing $\gamma=\boldsymbol{\gamma}(\tau)$ where $\tau=t / \varepsilon$, and $0<\varepsilon \ll 1$ is a small, nonrandom parameter. The meaning of this is as follows: as the "natural" time $t$ changes by a typical amount $\Delta t, \gamma(\tau)$ fluctuates considerably, since it experiences an elapsed time $\Delta \tau=\Delta t / \varepsilon$ which is large when $\varepsilon$ is small. Equivalently, an ordinary change of time $\Delta \tau$ on the time scale of the random phenomena will be a short time interval $\Delta t=\varepsilon \Delta \tau$ from the point of view of the rest of the system. Now if on its own fast time scale $\tau$ the process $\gamma(\tau)$ has an autocorrelation which dies out eventually, this will appear to happen very rapidly on the time scale of the rest of the system. Thus $\boldsymbol{\gamma}$ will be much like "white noise".

The parameter $\varepsilon$ may be estimated as a typical time for a random change in the environment divided by a typical time for a change in the populations. It must be small for application of the method. However, an exact value for $\varepsilon$ need not be known; the approximation used will combine $\varepsilon$ with other parameters in such a way that only more easily measured combinations must be specified.

We write $\mathbf{X}^{\varepsilon}(t)$ to denote the dependence of the population vector on $\varepsilon$. Then the stochastic modification of (2.1.1) is

$$
\frac{d}{d t} \mathbf{X}^{\varepsilon}(t)=\mathbf{F}\left(t, t / \varepsilon, \mathbf{X}^{\varepsilon}(t) ; \gamma(t / \varepsilon)\right),
$$

where $\gamma(t / \varepsilon)$ is the rapidly-varying random process. Equation (2.1.2) allows also for deterministic changes in the environment both on the natural time scale $t$ and on the fast time $t / \varepsilon$.

Using theoretical results of Khasminskii [5] and the author [12], we will exploit the disparity of time scales to get a two term perturbation approximation to $\mathbf{X}^{\varepsilon}(t)$. A derivation of the method is deferred until $\S 4$, and we merely describe the procedure here.

The first term of the approximation corresponds roughly to the mean value of $\mathbf{X}^{\varepsilon}(t)$, but not exactly-rather than averaging the unknown $\mathbf{X}^{\varepsilon}(t)$, we average the known function $\mathbf{F}$. The rapid fluctuations allow this averaging to be done not only in the probabilistic sense of taking expectations, but also in the sense of time averaging over the fast time variation of $\mathbf{F}$. Accordingly we define the simpler, 
averaged function $\mathbf{F}(t, \mathbf{x})$, for fixed values of $t$ and $\mathbf{x}$, by

$$
\overline{\mathbf{F}}(t, \mathbf{x})=\lim _{T \rightarrow \infty} \frac{1}{T} \int_{0}^{T} E[\mathbf{F}(t, \tau, \mathbf{x} ; \gamma(\tau))] d \tau .
$$

Then we formulate the following much simpler equation for the first approximation $\mathbf{X}^{0}(t)$ :

$$
\frac{d}{d t} \mathbf{X}^{0}(t)=\overline{\mathbf{F}}\left(t, \mathbf{X}^{0}(t)\right)
$$

The initial condition for $\mathbf{X}^{0}$ is the same as that for $\mathbf{X}^{\varepsilon}$. Equation (2.1.4) is completely deterministic and has only one time scale. It corresponds to a deterministic theory of interacting species with the interaction dynamics specified by $\overline{\mathbf{F}}$. Its solution $\mathbf{X}^{0}(t)$ determines the gross behavior of the stochastic population vector $\mathbf{X}^{\varepsilon}(t)$.

Equations for the approximate fluctuations about $\mathbf{X}^{0}(t)$ may be found by using the perturbation expansion

$$
\mathbf{X}^{\varepsilon}(t) \sim \mathbf{X}^{0}(t)+\sqrt{\varepsilon} \mathbf{Y}^{0}(t)
$$

Here $\mathbf{Y}^{0}(t)$ is a Gaussian random process which satisfies a linear equation given below. To write it, we compute the matrix of partial derivatives of the averaged function $\overline{\mathbf{F}}$, and evaluate it along the deterministic trajectory. That is, we define

$$
C_{i j}(t)=\frac{\partial \bar{F}_{i}}{\partial X_{j}}\left(t, \mathbf{X}^{0}(t)\right)
$$

Then $\mathbf{Y}^{0}$ satisfies the equation

$$
\frac{d}{d t} \mathbf{Y}^{0}(t)=C(t) \mathbf{Y}^{0}(t)+\mathbf{W}(t)
$$

where the initial condition is $\mathbf{Y}^{0}(0)=0$. Here $\mathbf{W}(t)$ is a Gaussian white noise with its covariance equal to the averaged covariance, $A$, of $\mathbf{F}$ evaluated along $X^{0}(t)$.

To compute $A$ we calculate for fixed $t$ and $\mathbf{x}$ the covariance of $\mathbf{F}$ with the fast time again averaged out. Thus

$$
\begin{array}{r}
A_{i j}(t, \mathbf{x}) \\
=\lim _{T \rightarrow \infty} \frac{1}{T} \int_{0}^{T} d \tau_{1} \int_{0}^{T} d \tau_{2} E\left\{\left[F_{i}\left(t, \tau_{1}, \mathbf{x} ; \gamma\left(\tau_{1}\right)\right)-E\left\{F_{i}\left(t, \tau_{1}, \mathbf{x} ; \gamma\left(\tau_{1}\right)\right)\right\}\right]\right. \\
\left.\left[F_{j}\left(t, \tau_{2}, \mathbf{x} ; \gamma\left(\tau_{2}\right)\right)-E\left\{F_{j}\left(t, \tau_{2}, \mathbf{x} ; \gamma\left(\tau_{2}\right)\right)\right\}\right]\right\} .
\end{array}
$$

With the abbreviation $A(t) \equiv A\left(t, \mathbf{X}^{0}(t)\right)$, the covariance of $\mathbf{W}(t)$ is

$$
\begin{aligned}
\operatorname{Cov}\left(\mathbf{W}(t), \mathbf{W}\left(t^{\prime}\right)\right) & =E\left[\mathbf{W}(t) \mathbf{W}^{T}\left(t^{\prime}\right)\right] \\
& =A(t) \delta\left(t-t^{\prime}\right) .
\end{aligned}
$$

Equation (2.1.7) may be recognized as a sort of stochastic variational equation for a linearization of the solution about $\mathbf{X}^{0}(t)$. 
The solution of (2.1.7) can be written explicitly in terms of the solution of the following system of linear deterministic equations:

$$
\frac{d}{d t} Y(t)=C(t) Y(t), \quad Y(0)=I .
$$

Then

$$
\mathbf{Y}^{0}(t)=\mathbf{Y}(t) \int_{0}^{t} \mathbf{Y}^{-1}(s) W(s) d .
$$

Since $Y^{0}$ is Gaussian, with mean zero, it is completely characterized by its covariance. This is computed as

$$
\begin{aligned}
\operatorname{Cov} & \left(\mathbf{Y}^{0}(t), \mathbf{Y}^{0}\left(t+t^{\prime}\right)\right)=E\left[\mathbf{Y}^{0}(t) \mathbf{Y}^{0^{T}}(t+\tau)\right] \\
& =E \mathbf{Y}(t) \int_{0}^{t} d s_{1} \int^{t+t^{\prime}} d s_{2} Y^{-1}\left(s_{1}\right) \mathbf{W}\left(s_{1}\right) \mathbf{W}^{T}\left(s_{2}\right) Y^{-1 T}\left(s_{2}\right) Y^{T}\left(t+t^{\prime}\right)
\end{aligned}
$$

By using (2.1.9) in the above equation, we obtain

$$
\operatorname{Cov}\left(\mathbf{Y}^{0}(t), \mathbf{Y}^{0}\left(t+t^{\prime}\right)\right)=Y(t) \int_{0}^{t} Y^{-1}(s) A(s) Y^{-1 T}(s) d s Y^{T}\left(t+t^{\prime}\right)
$$

Of particular interest in assessing the strength of the fluctuations is $D(t)$, the covariance at one instant of time, defined by

$$
\begin{aligned}
D(t) & =\operatorname{Cov}\left(\mathbf{Y}^{0}(t), \mathbf{Y}^{0}(t)\right) . \\
& =Y(t) \int_{0}^{t} Y^{-1}(s) A(s) Y^{-1 T}(s) d s Y^{T}(t)
\end{aligned}
$$

By setting $t^{\prime}=0$ in (2.1.12) differentiating the equation, and using (2.1.10) we find the formula

$$
\begin{aligned}
\frac{d}{d t} D(t) & =C(t) D(t)+D(t) C^{T}(t)+A(t), \\
D(0) & =0
\end{aligned}
$$

Although white noise was not assumed in the original equations, (2.1.14) agrees with an approximate result for white noise models (C. F. Ludwig [6]).

2.2. Point equilibria without delay. We shall now apply the preceding results to the special case of a system of species randomly perturbed by the environment from a point equilibrium. We suppose that the stochastic equations are of the form

$$
\frac{d}{d t} \mathbf{X}^{\varepsilon}(t)=\mathbf{F}\left(X^{\varepsilon}(t) ; \gamma(t / \varepsilon)\right)
$$

We assume that the deterministic system

$$
\frac{d}{d t} \mathbf{X}^{0}(t)=\overline{\mathbf{F}}\left(\mathbf{X}^{0}(t)\right)
$$


(where $\overline{\mathbf{F}}$ is computed by equation (2.1.3)) has a stable point equilibrium at some point $\overline{\mathbf{X}}$, i.e., $\overline{\mathbf{F}}(\mathbf{X})=0$. The problem is to compute the distribution of the random fluctuations about $\overline{\mathbf{X}}$.

For simplicity, we assume for the present that the populations are initially at equilibrium, $\mathbf{X}^{\varepsilon}(0)=\overline{\mathbf{X}}$. Then the deterministic trajectory remains at $\overline{\mathbf{X}}$ for all time, and the solution of (2.2.2) is

$$
\mathbf{X}^{0}(t)=\overline{\mathbf{X}} \text {. }
$$

Thus $C\left(\mathbf{X}^{0}(t)\right)=\partial \overline{\mathbf{F}}(\bar{X}) / \partial \mathbf{X}$ and $A\left(\mathbf{X}^{0}(t)\right)=A(\bar{X})$ are constant matrices.

In this context of a point equilibrium, $C$ has been called by Vandermeer [10] and others the "community matrix". It plays a fundamental role in the deterministic theory: the system is linearly stable if the eigenvalues of $C$ have negative real parts. We assume that this is the case, and calculate the correlation matrix of the Gaussian perturbation $Y^{0}(t)$.

Equation (2.1.10) now takes the form

$$
\frac{d}{d t} \mathbf{Y}(t)=C \mathbf{Y}(t), \quad Y(0)=I
$$

Its solution is

$$
\mathbf{Y}(t)=e^{C t}
$$

From (2.1.13) and (2.2.3) we get

This simplifies to

$$
\operatorname{Cov}\left(\mathbf{Y}^{0}(t), \mathbf{Y}^{0}\left(t+t^{\prime}\right)\right)=e^{C t} \int_{0}^{t} e^{-C s} A e^{-C T_{s}} d s e^{C T\left(t+t^{\prime}\right)}
$$

$$
\operatorname{Cov}\left(\mathbf{Y}^{0}(t), Y^{0}\left(t+t^{\prime}\right)\right)=\int_{0}^{t} e^{C s} A e^{C^{T} s} d s e^{C^{T_{t^{\prime}}}}
$$

Since the eigenvalues of $C$ have negative real parts, the factor $e^{C^{T} t^{\prime}}$ decays exponentially with increasing $t^{\prime}$. Thus correlations of the process at two instants of time die out exponentially in the time separation, and the exponential rate constants are the eigenvalues of $C$. Roughly, these are the rates at which the components of the fluctuations forget their former values.

Although the approximation is not valid for infinite observation time (see $\S 1$ ), the covariance matrix

$$
D(t)=\int_{0}^{t} e^{C s} A e^{C^{T}} d s
$$

converges to a limit $D(\infty)$ exponentially in $t$. This approach will be very rapid, especially if the deterministic equation is very stable, i.e., if the eigenvalues of $C$ have large negative real parts. Then the limit $D(\infty)$ will be established early and will continue for the remainder of the fixed, finite observation time.

We now differentiate, to obtain

$$
\frac{d}{d t} D(t)=e^{C t} A e^{C^{T} t} \rightarrow 0 \quad \text { as } t \rightarrow \infty
$$


Upon substituting this into the equation

$$
\frac{d}{d t} D(t)=C D(t)+D(t) C^{T}+A
$$

we obtain

$$
0=C D(\infty)+D(\infty) C^{T}+A
$$

This is a system of linear algebraic equations for $D(\infty)$. It has also been obtained by R. May [8] for point equilibria perturbed by a white noise environment. He has found good agreement of the solution with results of Monte Carlo simulations, although for very long times the approximation is somewhat degraded.

We shall now describe the general behavior of the solution more graphically. The contour lines of the Gaussian probability cloud about $\overline{\mathbf{X}}$ are ellipsoids determined by the covariance matrix $D(t)$. These ellipsoids expand in time as uncertainty in the size of the populations increases. If the deterministic system is linearly stable, however, this uncertainty quickly reaches a maximum, as the ellipsoids converge exponentially to the limiting ellipsoid determined by $D(\infty)$. The rate of convergence is governed by exponential rate constants which are sums of eigenvalues of the community matrix $C$.

We note that these formulas depend only on $\overline{\mathbf{X}}, C, \varepsilon$, and $A$. We now show that they in fact depend only on $\overline{\mathbf{X}}, C$, and the product $\varepsilon \cdot A$.

Since the approximation is $\mathbf{X}^{\varepsilon} \sim \mathbf{X}^{0}+\sqrt{\varepsilon} \mathbf{Y}^{0}$, the fluctuations are $\mathbf{X}^{\varepsilon}-\mathbf{X}^{0} \sim$ $\sqrt{\varepsilon} \mathbf{Y}^{0}$. This is Gaussian, mean zero, and is entirely specified by its covariance. From (2.1.12),

$$
\begin{aligned}
\operatorname{Cov}\left(\sqrt{\varepsilon} \mathbf{Y}^{0}(t), \sqrt{\varepsilon} \mathbf{Y}^{0}\left(t+t^{\prime}\right)\right)=\varepsilon \operatorname{Cov}\left(\mathbf{Y}^{0}(t), \mathbf{Y}^{0}\left(t+t^{\prime}\right)\right) \\
=Y(t) \int_{0}^{t} Y^{-1}(s)[\varepsilon A] Y^{-1 T}(s) d s Y^{T}\left(t+t^{\prime}\right) .
\end{aligned}
$$

Thus in computing the fluctuations $\mathbf{X}^{\varepsilon}-\mathbf{X}^{0}, \varepsilon$ can be absorbed into $A$ to give an "effective noise strength" $\varepsilon A$. (These considerations of absorbing $\varepsilon$ into $A$ apply also to all problems treated in this paper.)

Since the effective noise strength $\varepsilon A$ is small for small $\varepsilon$, our results will tend to agree with those of authors assuming small white noise. However, we have not assumed that the magnitude of the environmental fluctuations $\gamma(\tau)$ is small, only that the fluctuations are rapid.

The results for equilibrium point models therefore depend upon:

1. $\overline{\mathbf{X}}$, the position of the deterministic equilibrium.

2. $C$, the community matrix.

3. $\varepsilon A$, the "effective noise strength" at $\overline{\mathbf{X}}$.

All mathematical models agreeing in these particulars will give identical results to this order of approximation.

2.3. Two-species symmetric competition without delay. We now consider an example from the theory of niche overlap, of two similar species competing in a random environment. The equations for the populations $x(t)$ and $y(t)$ are 
assumed to be

$$
\frac{d}{d t}\left[\begin{array}{l}
x(t) \\
y(t)
\end{array}\right]=\left[\begin{array}{ll}
x(t) & \left(k+\gamma_{1}(t / \varepsilon)-x(t)-\alpha y(t)\right) \\
y(t) & \left(k+\gamma_{2}(t / \varepsilon)-y(t)-\alpha x(t)\right)
\end{array}\right] .
$$

The competition coefficient $\alpha, 0<\alpha<1$, measures the degree of competition. The deterministic carrying capacity $k$ (the same for both species) has been perturbed by the rapidly-fluctuating random processes $\gamma_{1}(\tau), \gamma_{2}(\tau)$, where $\tau=t / \varepsilon$.

We assume that time averages of the means of $\gamma_{1}(\tau), \gamma_{2}(\tau)$ are zero. Sufficient for this is $E\left[\gamma_{1}(\tau)\right]=E\left[\gamma_{2}(\tau)\right]=0$. However, the mean values may themselves fluctuate about zero provided that their time averages vanish sufficiently rapidly, e.g., $E\left[\gamma_{1}(\tau)\right]=\sin \tau$ or any other periodic function would be acceptable, and would change none of the following results.

We calculate

$$
\begin{aligned}
\overline{\mathbf{F}}(X) & =\lim _{T \rightarrow \infty} \frac{1}{T} \int_{0}^{T} E[F(X, \tau)] d \tau \\
& =\left[\begin{array}{ll}
x & (k-x-\alpha y) \\
y & (k-y-\alpha x)
\end{array}\right] .
\end{aligned}
$$

The deterministic equation is then

$$
\frac{d}{d t}\left[\begin{array}{l}
x^{0}(t) \\
y^{0}(t)
\end{array}\right]=\left[\begin{array}{ll}
x^{0}(t) & \left(k-x^{0}(t)-\alpha y^{0}(t)\right) \\
y^{0}(t) & \left(k-y^{0}(t)-\alpha x^{0}(t)\right)
\end{array}\right] .
$$

These are just the competition equations in a fixed environment. There are two equilibria, one at the origin, and one at

$$
\overline{\mathbf{X}}=\left[\begin{array}{c}
\frac{k}{1+\alpha} \\
\frac{k}{1+\alpha}
\end{array}\right]
$$

If the system starts at $\overline{\mathbf{X}}$ at time zero, we have $\mathbf{X}^{0}(t)=\overline{\mathbf{X}}$ for $t>0$.

The community matrix is

$$
C=\frac{\partial \overline{\mathbf{F}}}{\partial \mathbf{X}}(\overline{\mathbf{X}})=-\frac{k}{1+\alpha}\left[\begin{array}{cc}
1 & \alpha \\
\alpha & 1
\end{array}\right]
$$

From (2.3.1) and (2.3.2) we have

$$
\begin{gathered}
\mathbf{F}(\overline{\mathbf{X}}, \tau)-E[\mathbf{F}(\overline{\mathbf{X}}, \tau)]=\frac{k}{1+\alpha}\left[\begin{array}{l}
\gamma_{1}(\tau) \\
\gamma_{2}(\tau)
\end{array}\right] \\
A(\overline{\mathbf{X}})=\left(\frac{k}{1+\alpha}\right)^{2} \lim _{T \rightarrow \infty} \frac{1}{T} \int_{0}^{T} \int_{0}^{T} E\left[\begin{array}{ll}
\gamma_{1}\left(\tau_{1}\right) \gamma_{1}\left(\tau_{2}\right) & \gamma_{1}\left(\tau_{1}\right) \gamma_{2}\left(\tau_{2}\right) \\
\gamma_{2}\left(\tau_{1}\right) \gamma_{1}\left(\tau_{2}\right) & \gamma_{2}\left(\tau_{1}\right) \gamma_{2}\left(\tau_{2}\right)
\end{array}\right] d \tau_{1} d \tau_{2}
\end{gathered}
$$

The symmetric matrix $A(\overline{\mathbf{X}})$ depends upon just three noise parameters, calculated by time averaging the variances and correlations of $\gamma_{1}$ and $\gamma_{2}$. Thus it is not necessary to specify completely the statistics of $\gamma_{1}$ and $\gamma_{2}$, and the noise need not be known in detail. 
To preserve the symmetry of the competition, we assume here equal averaged noise variances $\sigma^{2}$ for the processes $\gamma_{1}$ and $\gamma_{2}$. Then we can write $A$ in the form

$$
A=\left(\frac{k}{1+\alpha}\right)^{2}\left[\begin{array}{cc}
\sigma^{2} & \sigma^{2} \rho \\
\sigma^{2} \rho & \sigma^{2}
\end{array}\right],
$$

where $\rho,-1 \leqq \rho \leqq+1$, is the time averaged cross correlation of $\gamma_{1}$ and $\gamma_{2}$.

Equations (2.3.4), (2.3.5), and (2.3.6), (2.1.14) are sufficient to determine the probability cloud of the fluctuations $Y^{0}(t)$. The results are most easily described by transforming to the principle coordinates of the matrices $C$ and $A$, a $45^{\circ}$ rotation of the $x, y$ coordinates about $\overline{\mathbf{X}}$. Accordingly, we define the rotation matrix

$$
P=\frac{1}{\sqrt{2}}\left[\begin{array}{rr}
1 & 1 \\
1 & -1
\end{array}\right]
$$

and the transformed matrices

$$
\begin{aligned}
& \hat{A}=P^{-1} A P=\left(\frac{k}{1+\alpha}\right)^{2} \sigma^{2}\left[\begin{array}{cc}
1+\rho & 0 \\
0 & 1-\rho
\end{array}\right], \\
& \hat{C}=P^{-1} C P=-\frac{k}{1+\alpha}\left[\begin{array}{cc}
1+\alpha & 0 \\
0 & 1-\alpha
\end{array}\right] .
\end{aligned}
$$

The eigenvalues of $C$ are evidently $-k$ and $-(1-\alpha) k /(1+\alpha)$. These determine the exponential rates of decay of the correlations of the population sizes at two separate instants of time. The rates are rapid except in the important case when $\alpha$ is close to one, and $-k(1-\alpha) /(1+\alpha)$ is close to zero. Then the second component of the fluctuations, the deviation from the $45^{\circ}$ line of equal population sizes, has a slow rate of forgetfulness. Thus only when competition is very high, does the system not readily forget which species is momentarily more numerous.

To determine the probability cloud at one instant of time, we calculate

$$
\begin{aligned}
\hat{D}(t) & =P^{-1} D(t) P=\int_{0}^{t} e^{\hat{C s}} \hat{A} e^{C^{T} s} d s \\
& =\frac{\sigma^{2} k}{2}\left[\begin{array}{cc}
\frac{(1+\rho)}{(1+\alpha)^{2}}\left(1-e^{-2 k t}\right) & 0 \\
0 & \frac{(1-\rho)}{\left(1-\alpha^{2}\right)}\left(1-\exp \left\{-\frac{2 k(1-\alpha) t}{(1+\alpha)}\right\}\right)
\end{array}\right]
\end{aligned}
$$

This converges exponentially to the limiting variance

$$
\hat{D}(\infty)=\frac{\sigma^{2} k}{2}\left[\begin{array}{cc}
\frac{1+\rho}{(1+\alpha)^{2}} & \\
0 & \frac{(1-\rho)}{\left(1-\alpha^{2}\right)}
\end{array}\right] .
$$

Again, the convergence is rapid except when $\alpha$ is close to 1 . In this case of high competition, the second component of $\hat{D}(\infty),\left(\sigma^{2} k / 2\right)(1-\rho) /\left(1-\alpha^{2}\right)$ is large. 
This indicates that higher competition will produce larger deviation from the line of equal population sizes.

This effect may be offset, however, if $\rho$ is also close to 1, i.e., if the random conditions encountered by the two species are highly correlated. Evidently, it is unlikely for one of the species to be substantially luckier than the other if their environments are substantially the same.

The limiting variance, (2.3.11), agrees with a result of May [8] for a white noise environment. In $\S 3.2$ we will generalize the equations of this section to study the effects of time-delayed interactions on this type of system.

2.4. Starting point near equilibrium. In the preceding analysis, it was assumed for simplicity that the system starts from the equilibrium point, that is, $\mathbf{X}^{\varepsilon}(0)=\overline{\mathbf{X}}$. Suppose instead that the populations are initially near $\overline{\mathbf{X}}$. Since the deterministic system is linearly stable, $\mathbf{X}^{0}(t)$ will converge to $\overline{\mathbf{X}}$ as time increases. This convergence will be exponential, with exponential rate constants that are the eigenvalues of $C$.

Now if a limiting variance $D(\infty)$ exists, then $(d / d t) D(t) \rightarrow 0$ as $t \rightarrow \infty$. Using $\mathbf{X}^{0}(t) \rightarrow \mathbf{X}$ as $t \rightarrow \infty$, we may take limits in the equation

$$
\frac{d}{d t} D(t)=C\left(\mathbf{X}^{0}(t)\right) D(t)+D(t) C^{T}\left(X^{0}(t)\right)+A\left(X^{0}(t)\right)
$$

to get

$$
0=C(\overline{\mathbf{X}}) D(\infty)+D(\infty) C^{T}(\overline{\mathbf{X}})+A(\overline{\mathbf{X}}) .
$$

This is the same set of algebraic equations that were obtained assuming $\overline{\mathbf{X}}$ as the starting point. Thus the limiting variance $D(\infty)$ is not affected.

In view of the exponential rate of convergence of the coefficients in (2.4.1), we expect the convergence of $D(t)$ to remain exponential. Indeed, this is intuitively expected because of the exponential rate of forgetfulness remarked upon earlier.

The effect of a near-equilibrium starting point is, then, to give rise to additional transient terms in the expression for $D(t)$. These have been computed explicitly for the example of the last section (see [13]), but the details will be omitted here. The extra terms are small initially, and decay exponentially at rates no slower than those determined by the eigenvalues of $C$.

If there is more than one stable equilibrium, our formulas describe the situation while the populations remain in the domain of attraction of one of them. They will, however, eventually escape this domain, although for small noise this will take a very long time. Problems of escape against a deterministic flow have been studied by Ventzel and Freidlin [11] and Ludwig [6].

2.5. The limit cycle. For many systems of species, the populations undergo regular cyclical oscillations. These have often been described mathematically by systems of ordinary differential equations whose solution is a stable limit cycle. Thus the situation is qualitatively different from that of the point equilibria already discussed.

We would expect the populations in a random environment to oscillate roughly in the way predicted by deterministic theory, but to fluctuate randomly 
from this moving equilibrium. These expectations have been confirmed by Monte Carlo simulations (see May [8]) assuming white noise for the environmental randomness. For large enough noise strengths, however, randomness can become the dominant effect, and the roughly cyclical behavior is destroyed.

For simplicity, we will analyze here the case of a limit cycle with only two species. The stochastic equations are assumed to be

$$
\frac{d}{d t} \mathbf{X}^{\varepsilon}(t)=F\left(\mathbf{X}^{\varepsilon}(t) ; \gamma(t / \varepsilon)\right), \quad X^{\varepsilon} \in R^{2} .
$$

It is assumed that the deterministic solution $\mathbf{X}^{0}(t)$ of the averaged equation

$$
\frac{d}{d t} \mathbf{X}^{0}(t)=\overline{\mathbf{F}}\left(\mathbf{X}^{0}(t)\right)
$$

is a stable limit cycle in the plane. Then, since the matrices $C(t)$ and $A(t)$ defined by (2.1.6) and (2.1.8) are obtained by evaluating certain functions of $\mathbf{x}$ along $\mathbf{X}^{0}(t)$, they will be periodic in time, with the same period, $\tau$, as the deterministic limit cycle.

To proceed with the general method, we must obtain a fundamental solution matrix of the equation

$$
\frac{d}{d t} Y(t)=C(t) Y(t)
$$

Floquet theory (see [2]) gives the following structure of the solution $Y(t)$ :

$$
Y(t)=\left[\begin{array}{ll}
P_{1}(t) & e^{\rho t} P_{2}(t) \\
Q_{1}(t) & e^{\rho t} Q_{2}(t)
\end{array}\right]
$$

where $P_{1}(t), P_{2}(t), Q_{1}(t), Q_{2}(t)$ are periodic in time with period $\tau$. Furthermore,

$$
\left[\begin{array}{l}
P_{1}(t) \\
Q_{1}(t)
\end{array}\right]=\frac{d}{d t} \mathbf{X}^{0}(t)=\left|\frac{d}{d t} \mathbf{X}^{0}(t)\right| \mathbf{T}(t),
$$

where $\mathbf{T}(t)$ is the unit tangent to the limit cycle at the point $\mathbf{X}^{0}(t)$. The constant $\rho$ is given by

$$
\rho=\frac{1}{\tau} \int_{0}^{\tau}[\operatorname{trace} C(s)] d s .
$$

With this choice of fundamental solution, we have

$$
\begin{aligned}
Y(t+\tau) & =Y(t) \Lambda, \\
\Lambda & =\left[\begin{array}{ll}
1 & 0 \\
0 & \lambda
\end{array}\right], \\
\lambda & =e^{\rho \tau} .
\end{aligned}
$$

Here $\lambda$, called the Floquet multiplier, must satisfy $0<\lambda<1$ for the limit cycle $\mathbf{X}^{0}(t)$ to be stable. 
We are interested in the covariance, $D(t)$ of the fluctuations. From equation

$$
D(t)=Y(t) \int_{0}^{t} Y^{-1}(s) A(s) Y^{-1 T}(s) d s Y^{T}(t)
$$

Define

$$
\begin{aligned}
& R(t)=Y(t) \int_{t}^{t+\tau} Y^{-1}(s) A(s) Y^{-1 T}(s) d s Y^{T}(t) \\
& H(t)=Y(t) \Lambda Y^{-1}(t)
\end{aligned}
$$

From (2.5.4), (2.5.5) and the periodicity of $A$, it may be verified that $R(t)$ and $H(t)$ are periodic of period $\tau$. Using this in (2.5.8), we obtain

$$
D(t+\tau)=H(t) D(t) H^{T}(t)+H(t) R(t) H^{T}(t) .
$$

By iterating this equation it may be obtained inductively that for $k$ a positive integer

$$
D(t+k \tau)=H^{k}(t) D(t) H^{T k}(t)+\sum_{l=1}^{k} H^{l}(t) R(t) H^{T l}(t) .
$$

Now consider the deterministic limit cycle as a set of points in the plane. The random quantities of interest are the distance of the populations from the closest point on the limit cycle, and the fluctuations in "phase", or angular position around the limit cycle. Thus the natural coordinates to use are the coordinates $(s, r)$, where $s$ is arc length along the limit cycle, and $r$ is perpendicular distance from it. The formulas above have been derived in Cartesian coordinates. However there is a change of variables result (see [13]) from which we may deduce from the Cartesian $D(t)$, the covariance $\hat{D}(t)$ which would have been obtained using another locally orthogonal coordinate system. Using $(s, r)$, the result is

$$
\hat{D}(t)=\left[\begin{array}{ll}
T^{T}(t) D(t) T(t) & T^{T}(t) D(t) N(t) \\
N^{t}(t) D(t) T(t) & N^{T}(t) D(t) N(t)
\end{array}\right]
$$

Here $T(t)$ and $N(t)$ are respectively the unit tangent and normal vectors at the point $\mathbf{X}^{0}(t)$.

We shall now investigate the various quadratic forms indicated in (2.5.12).

Now

$$
\begin{aligned}
N^{T} H^{k} & =\frac{1}{\left|\dot{X}^{0}\right|}\left[-Q_{1}, P_{1}\right]\left[\begin{array}{ll}
P_{1} & e^{\rho t} P_{2} \\
Q_{1} & e^{\rho t} Q_{2}
\end{array}\right]\left[\begin{array}{cc}
1 & 0 \\
0 & \lambda^{k}
\end{array}\right]\left[\begin{array}{cc}
e^{\rho t} Q_{2} & -e^{\rho t} P_{2} \\
-Q_{1} & P_{1}
\end{array}\right] \cdot \frac{e^{-\rho t}}{\left(P_{1} Q_{1}-P_{2} Q_{1}\right)} \\
& =\lambda^{k} N^{T}
\end{aligned}
$$

Since $N(t)$ is periodic of period $\tau$, we obtain from (2.5.11), and the condition 
$0<\lambda<1$

$$
\begin{aligned}
& N^{T}(t+k \tau) D(t+k \tau) N(t+k \tau) \\
& =\lambda^{2 k} N^{T}(t) D(t) N(t)+\sum_{l=1}^{k} \lambda^{2 l} N^{T}(t) R(t) N(t) \\
& \quad \underset{k \rightarrow \infty}{\rightarrow} \frac{\lambda^{2}}{1-\lambda^{2}} N^{T}(t) R(t) N(t) .
\end{aligned}
$$

Therefore the quadratic form $N^{T} D N$ converges through integral multiples of the period to a limiting value, and the convergence is geometric in the square of the Floquet multiplier $\lambda$.

The unit vector $B(t)$ orthogonal to the second column of $Y(t)$ is

$$
B(t)=\frac{1}{\sqrt{P_{2}^{2}+Q_{2}^{2}}}\left[\begin{array}{c}
-Q_{2} \\
P_{2}
\end{array}\right] .
$$

An analysis for $B(t)$ similar to that for $N(t)$ gives that

$$
B^{T}(t) H^{k}(t)=B^{T}(t)
$$

and that

$$
B^{T}(t+k \tau) D(t+k \tau) B(t+k \tau)=B^{T}(t) D(t) B(t)+k B^{T}(t) R(t) B(t) .
$$

Therefore, the quadratic form $B^{T} D B$ diverges linearly in $k$. Since the tangent $T(t)$ has a component in this direction, the same is true for the quadratic form $T^{T} D T$.

From the above discussion, it is evident that the variance in the "phase" $s$ grows linearly with the number of revolutions of the deterministic trajectory $\mathbf{X}^{0}(t)$. This is analogous to the deterministic situation where a particle moved slightly off the limit cycle will in time get increasingly out of phase through having its period slightly altered.

We may consider, however, the one-dimensional process $r(t)$, the random distance from the limit cycle at time $t$. It is approximately Gaussian, has mean zero, and variance $N^{T}(t) D(t) N(t)$ which converges to a limiting variance as $t=t_{0}+k \tau$ goes to infinity through integer values of $k$. To visualize this, we may plot on the same graph as $\mathbf{X}^{0}(t)$ the one-standard-deviation curves $X^{0}(t) \pm$ $\sqrt{N^{T}(t) D(t) N(t)} \mathbf{N}(t)$ of the process $\mathbf{X}^{0}(t)+r(t) \mathbf{N}(t)$. These wind away from the limit cycle $\mathbf{X}^{0}(t)$, and as the number of revolutions increases, they converge to the inner and outer boundaries of a limiting tube about the deterministic trajectory.

This has been done for the following predator-prey model proposed by $R$. May [8]:

$$
\begin{aligned}
& \frac{d N_{1}}{d t}=\left(r_{0}+\gamma\right) N_{1}\left(1-\frac{N_{1}}{K}\right)-a N_{2}\left(1-e^{-c N_{1}}\right), \\
& \frac{d N_{2}}{d t}=-s N_{2}+b N_{2}\left(1-e^{-c N_{1}}\right) .
\end{aligned}
$$

Figure 1 reproduces from May's paper a typical run of his Monte Carlo simulations for this model when $\gamma=\gamma(t)$ is white noise with variance $\sigma^{2}$. Plotted are 


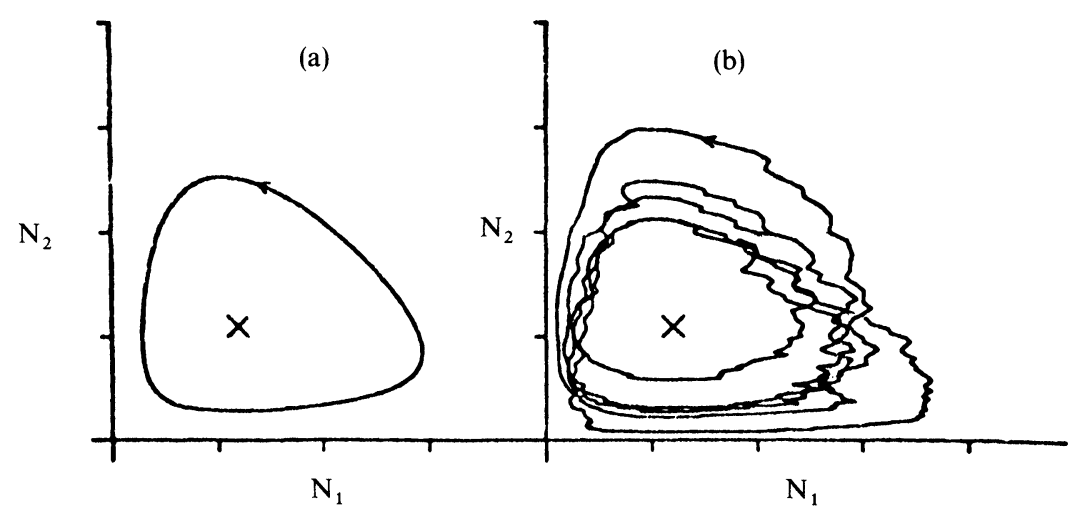

Fig. 1. From R. May [8].

dimensionless variables $x=c N_{1}, y=(c a / r) N_{2}$ for the choice of parameters $b / s=1.5, c K=4, r_{0}=s, \sigma^{2} / r_{0}=.1$. The graph conveys, according to May, "a good impression of the donut-shaped probability cloud which describes the system".

Figure 2 is our calculation of the one standard deviation tube about the limit cycle as described above. The choice of parameters was the same as May's except that we took $\gamma=\gamma(t / \varepsilon)$ to be a general process consistent with our theory, with $\varepsilon$ small, and $\varepsilon \sigma^{2} / r_{0}=.1$. After numerically integrating the equations of the deterministic limit cycle, the covariance matrix was obtained by numerically integrating

$$
\frac{d}{d t} D(t)=C\left(\mathbf{X}^{0}(t)\right) D(t)+D(t) C^{T}\left(\mathbf{X}^{0}(t)\right)+A\left(\mathbf{X}^{0}(t)\right)
$$

The agreement of our calculations with May's simulations appears at least qualitatively good. The simulated population point tends to wander farther from the limit cycle where our tube is relatively wide, and is more tightly bound where the tube is narrow.

\section{PART II}

\section{Random environments and time delays.}

3.1. Extension of the method. The method is little changed if the species interactions involve time lags. Then the interaction function $\mathbf{F}$ depends not only on $\mathbf{X}^{\varepsilon}(t)$, but on the vectors $X^{\varepsilon}\left(t-\delta_{1}\right), \cdots, X^{\varepsilon}\left(t-\delta_{N}\right)$ of population sizes at previous times, where $0 \equiv \delta_{0}<\delta_{1}<\cdots<\delta_{N}$. The equations are of the form

$$
\begin{aligned}
& \frac{d}{d t} \mathbf{X}^{\varepsilon}(t)=\mathbf{F}\left(t, t / \varepsilon, \mathbf{X}^{\varepsilon}(t), \mathbf{X}^{\varepsilon}\left(t-\delta_{1}\right), \cdots, \mathbf{X}^{\varepsilon}\left(t-\delta_{N}\right) ; \gamma(t / \varepsilon)\right) . \\
& X^{\varepsilon}(t) \text { given for } t \in\left[-\delta_{N}, 0\right] .
\end{aligned}
$$

As before, it is assumed that the environmental randomness $\gamma(\tau)$ is on a fast time scale $\tau=t / \varepsilon$, and that deterministic changes in the environment can be on both the slow and fast time scales.

It is still possible to find an approximation of the form

$$
\mathbf{X}^{\varepsilon}(t) \sim \mathbf{X}^{0}(t)+\sqrt{\varepsilon} \mathbf{Y}^{0}(\dot{t})
$$




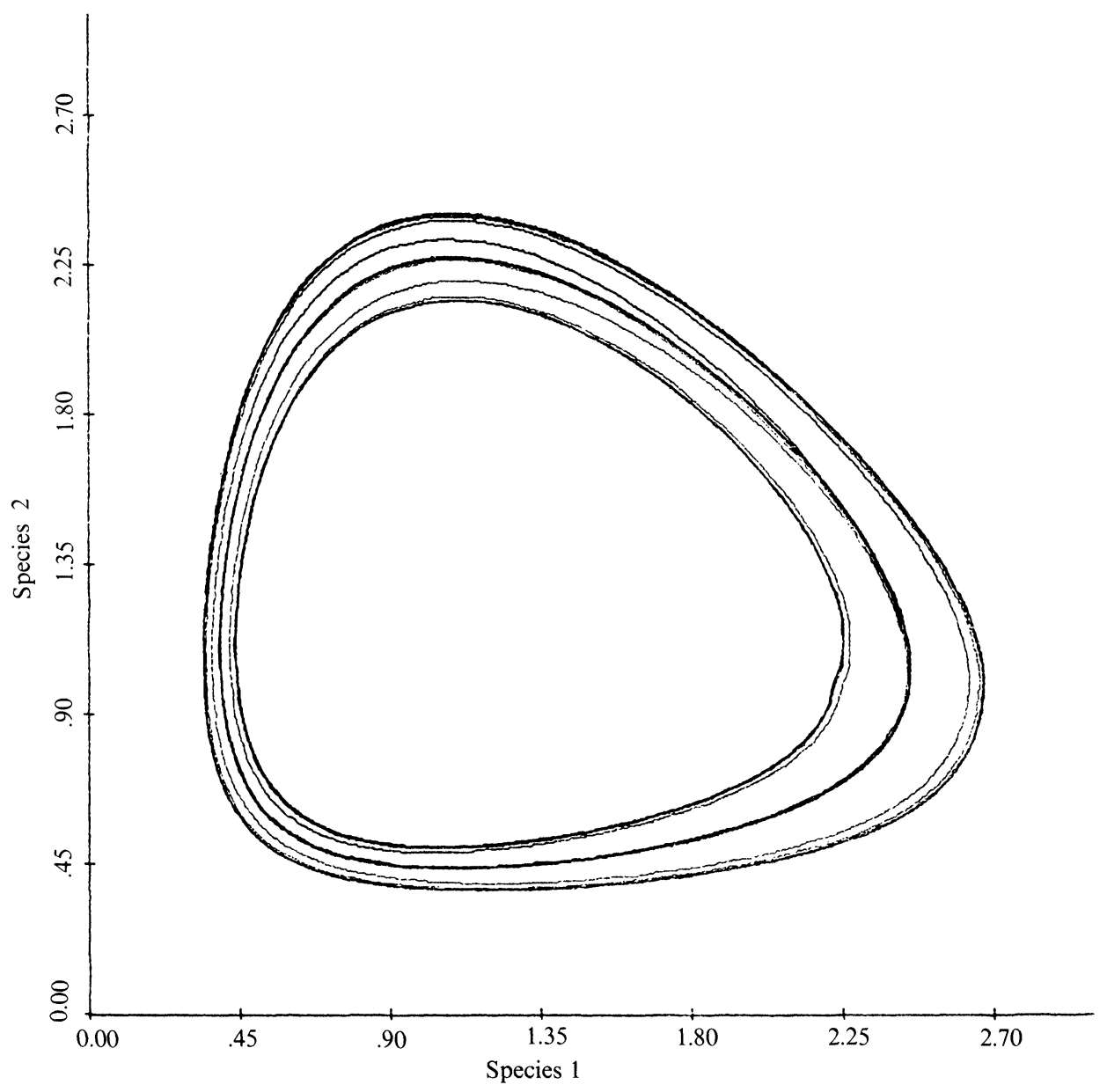

FIG. 2

where $X^{0}$ is deterministic and $Y^{0}$ Gaussian. We define for fixed $t$ and fixed vectors $\mathbf{x}_{0}, \mathbf{x}_{1}, \cdots, \mathbf{x}_{N}$, the averaged function

(3.1.3) $\quad \overline{\mathbf{F}}\left(t, \mathbf{x}_{0} \mathbf{x}_{1}, \cdots, x_{N}\right)=\lim _{T \rightarrow \infty} \frac{1}{T} \int_{0}^{T} E\left[\mathbf{F}\left(t, \tau, \mathbf{x}_{0}, \cdots, \mathbf{x}_{N} ; \gamma(\tau)\right)\right] d \tau$.

The first approximation $X^{0}(t)$, satisfies the nonrandom equation

$$
\frac{d}{d t} \mathbf{X}^{0}(t)=\overline{\mathbf{F}}\left(t, \mathbf{X}^{0}(t), \mathbf{X}^{0}\left(t-\delta_{1}\right), \cdots, \mathbf{X}^{0}\left(t-\delta_{N}\right)\right)
$$

Initial conditions for $X^{0}$ are the same as those for $\mathbf{X}^{\varepsilon}$.

We define the matrices $C_{k}$ by

$$
C_{k}\left(t, \mathbf{x}_{0}, \cdots, \mathbf{x}_{N}\right)=\frac{\partial \overline{\mathbf{F}}}{\partial \mathbf{x}_{k}}\left(t, \mathbf{x}_{0}, \cdots, \mathbf{x}_{N}\right), \quad k=0, \cdots, N .
$$


We define the averaged covariance matrix $A$ by

$$
\begin{aligned}
& A_{i j}\left(t, x_{0}, \cdots, x_{N}\right) \\
&=\lim _{T \rightarrow \infty} \frac{1}{T} \int_{0}^{T} \int_{0}^{T} E\left\{\left[F_{i}\left(t, \tau_{1}, \mathbf{x}_{0}, \cdots, \mathbf{x}_{N} ; \gamma\left(\tau_{1}\right)\right)\right.\right. \\
&-E\left\{F_{i}\left(t, \tau_{1}, \mathbf{x}_{0}, \cdots, \mathbf{x}_{N} ; \gamma\left(\tau_{1}\right)\right)\right\} \\
& \cdot\left[F_{j}\left(t, \tau_{2}, \mathbf{x}_{0}, \cdots, \mathbf{x}_{N} ; \gamma\left(\tau_{2}\right)\right)\right. \\
&\left.\left.-E\left\{F_{j}\left(t, \tau_{2}, \mathbf{x}_{0}, \cdots, \mathbf{x}_{N} ; \boldsymbol{\gamma}\left(\tau_{2}\right)\right)\right\}\right]\right\} d \tau_{1} d \tau_{2} .
\end{aligned}
$$

We abbreviate

$$
\begin{aligned}
C_{k}(t) & \equiv C_{k}\left(t, \mathbf{X}^{0}(t), \mathbf{X}^{0}\left(\mathrm{t}-\delta_{1}\right), \cdots, \mathbf{X}^{0}\left(t-\delta_{N}\right)\right), \\
A(t) & =A\left(t, \mathbf{X}^{0}(\mathrm{t}), \mathbf{X}^{0}\left(t-\delta_{1}\right), \cdots, \mathbf{X}^{0}\left(t-\delta_{N}\right)\right) .
\end{aligned}
$$

Then $\mathbf{Y}^{0}(t)$ satisfies the linear stochastic delay-differential equation

$$
\begin{aligned}
\frac{d}{d t} Y^{0}(t) & =W(t)+\sum_{k=0}^{N} C_{k}(t) Y^{0}\left(t-\delta_{k}\right), \\
Y^{0}(t) & \equiv 0 \quad \text { for } t<0 .
\end{aligned}
$$

Here $W(t)$ is a Gaussian white noise with covariance $A(t) \delta\left(t-t^{\prime}\right)$.

Equation (3.1.7) can be solved if we can find the fundamental solution matrix $Y(t, s)$ which satisfies the deterministic equations

$$
\begin{array}{cc}
\frac{d}{d t} Y(t, s)=\sum_{k=0}^{N} C_{k}(t) Y\left(t-\delta_{k}, s\right), & t>s \\
Y(t, s)=0, & t>s \\
Y(s, s)=I . &
\end{array}
$$

In terms of $Y(t, s)$, the solution of (3.1.7) may be written

$$
\mathbf{Y}^{0}(t)=\int_{0}^{t} Y(t, s) W(s) d s
$$

The covariance of the Gaussian process $\mathbf{Y}^{0}(t)$ is then

$$
\operatorname{Cov}\left(Y^{0}(t), Y^{0}\left(t+t^{\prime}\right)\right)=\int_{0}^{t} Y(t, s) A(s) Y^{T}\left(t+t^{\prime}, s\right) d s
$$

3.2. Equilibrium points for systems with time lag. We consider the case when the averaged interaction function $\overline{\mathbf{F}}$, calculated from (3.1.3) has a stable equilibrium point. The deterministic equation for $\mathbf{X}^{0}(t)$ is of the form

$$
\frac{d}{d t} \mathbf{X}^{0}(t)=\overline{\mathbf{F}}\left(\mathbf{X}^{0}(t), \mathbf{X}^{0}\left(t-\delta_{1}\right), \cdots, \mathbf{X}^{0}\left(t-\delta_{N}\right)\right)
$$

It is assumed that there is an equilibrium point $\overline{\mathbf{X}}$ such that $\overline{\mathbf{F}}(\overline{\mathbf{X}}, \overline{\mathbf{X}}, \cdots, \overline{\mathbf{X}})=0$. It is also assumed that $\overline{\mathbf{X}}$ is stable, so that $\mathbf{X}^{0}(t) \rightarrow \bar{X}$ if the initial conditions 
$\boldsymbol{\phi}(t)=\mathbf{X}^{0}(t)$ in $\left[-\delta_{N}, 0\right]$ are sufficiently close to $\overline{\mathbf{X}}$. Such equations have an extensive literature (see e.g. Halany [4], Pinney [9]), so there will be little discussion of them here. In general, if the nondelay system (set $\delta_{i}=0, i=$ $1, \cdots, N$, in (3.2.1)) is stable, then so is the delay system for small enough delays. Longer time lags may, however, bring on instability, although some systems (notably that of the next section) are immune to this.

For simplicity, we will assume $\phi(t) \equiv \overline{\mathbf{X}}$ for $t \in\left[-\delta_{N}, 0\right]$, so that $\mathbf{X}^{0}(t)=\overline{\mathbf{X}}$ for $t>0$. The matrices

$$
C_{k}=\frac{\partial \overline{\mathbf{F}}}{\partial \overline{\mathbf{X}}_{k}}(\overline{\mathbf{X}}, \cdots, \overline{\mathbf{X}}), \quad k=0,1, \cdots, N,
$$

are constant as is the matrix

$$
A(\overline{\mathbf{X}}, \overline{\mathbf{X}}, \cdots, \overline{\mathbf{X}})
$$

calculated from (3.1.6).

Since the coefficients in (3.1.8) do not depend on time, we have the identity

$$
Y(t, s)=Y(t-s, 0) .
$$

Then $Y(t) \equiv Y(t, 0)$ satisfies the equation

$$
\begin{array}{rlrl}
\frac{d}{d t} Y(t) & =\sum_{k=0}^{N} C_{k} Y\left(t-\delta_{k}\right), & t>0, \\
Y(t) & \equiv 0 \text { for } t<0, \\
Y(0) & =I .
\end{array}
$$

Equation (3.2.2) can be solved by Laplace transforms (see e.g. Pinney [9]). Important for this is the characteristic function $f(s)$ defined by

$$
f(s)=\operatorname{det}\left[s I-\sum_{k=0}^{N} C_{k} e^{-\delta_{k} s}\right] .
$$

The character of the solution is determined by the roots $s=\lambda_{k}$ of the transcendental equation

$$
f(s)=0 .
$$

Equation (3.2.4) has, in general, an infinite number of complex roots. We require that all these roots have negative real parts; this is the same condition as for linear asymptotic stability of the nonlinear equation (3.2.1). These roots $\lambda_{k}$ play the same role in the time-lag theory of equilibrium points as did the eigenvalues of the community matrix previously.

The roots $\lambda_{k}$ are written in order of decreasing real parts. If the roots are simple then the solution of (3.2.2) is

$$
Y(t)=\sum_{k=1}^{N} e^{\lambda_{k} t} Y_{k}
$$


The "residue" matrices $Y_{k}$ are calculated as

$$
\begin{aligned}
Y_{k} & =\lim _{s \rightarrow \lambda_{k}}\left(s-\lambda_{k}\right)\left[s I-\sum_{m=0}^{N} C_{m} e^{-\delta_{m} s}\right]^{-1} \\
& =\frac{1}{f^{\prime}\left(\lambda_{k}\right)}\left[\text { Cofactor }\left(\lambda_{k} I-\sum_{m=0}^{N} C_{m} e^{-\delta_{m} \lambda_{k}}\right)\right]^{T}
\end{aligned}
$$

Equation (3.1.9) then becomes

$$
\begin{aligned}
\operatorname{Cov}\left(Y^{0}(t), Y^{0}\left(t+t^{\prime}\right)\right) & =\int_{0}^{t} Y(s) A Y^{T}\left(s+t^{\prime}\right) d s \\
& =\sum_{k=1}^{\infty} \sum_{l=1}^{\infty} e^{\lambda_{l} t^{\prime}} \frac{\left(1-e^{\left(\lambda_{k}+\lambda_{l}\right) t}\right)}{\left(\lambda_{k}+\lambda_{l}\right)} Y_{k} A Y_{l}^{T} .
\end{aligned}
$$

From (3.2.7) we see that the correlations of the fluctuation process $Y^{0}(t)$ at different instants of time go to zero exponentially in the time separation $t^{\prime}$. Each term in the sum (3.2.7) vanishes with a rate constant $\lambda_{l}$. Thus the fluctuations forget their former position at an exponential rate no slower than that determined by the characteristic root $\lambda_{1}$ of largest real part.

The covariance of $Y^{0}$ is

$$
D(t)=\sum_{k=1}^{\infty} \sum_{l=1}^{\infty} \frac{\left(1-e^{\left(\lambda_{k}+\lambda_{l}\right) t}\right)}{\left(\lambda_{k}+\lambda_{l}\right)} Y_{k} A Y_{. l}^{T}
$$

This converges to the limiting covariance

$$
D(\infty)=\sum_{k=1}^{\infty} \sum_{l=1}^{\infty} \frac{1}{\left(\lambda_{k}+\lambda_{l}\right)} Y_{k} A Y_{l}^{T}
$$

The convergence is exponential, at a rate determined by sums of the characteristic roots.

To summarize, the general behavior is much the same as in the nondelay case ( $\$ 2.2$ ), with the characteristic roots $\lambda_{k}$ taking the place of the eigenvalues of the community matrix. The calculations, however, involve evaluating infinite sums. That this may be done explicitly, at least in some cases, is illustrated by the next example.

\subsection{Two-species symmetric competition with time-delayed} interactions. We now consider the following extension of the model of $\S 2.3$ :

$$
\frac{d}{d t}\left[\begin{array}{l}
x(t) \\
y(t)
\end{array}\right]=\left[\begin{array}{l}
x(t)\left(k+\gamma_{1}(t / \varepsilon)-x(t)-\alpha y(t-\delta)\right) \\
y(t)\left(k+\gamma_{2}(t / \varepsilon)-y(t)-\alpha x(t-\delta)\right)
\end{array}\right]
$$

Here a time lag $\delta>0$ has been introduced into the interspecies interaction terms. The assumptions on the rapidly-varying environmental processes $\gamma_{1}(\tau), \gamma_{2}(\tau)$ are the same as in $\S 2.3$. We assume $0 \leqq \alpha<1$.

The averaged equations for $\mathbf{X}^{0}(t)$ are

$$
\frac{d}{d t}\left[\begin{array}{l}
x^{0}(t) \\
y^{0}(t)
\end{array}\right]=\left[\begin{array}{l}
x^{0}(t)\left(k-x^{0}(t)-\alpha y^{0}(t-\delta)\right) \\
y^{0}(t)\left(k-y^{0}(t)-\alpha x^{0}(t-\delta)\right)
\end{array}\right]
$$


The equilibrium point is at

$$
\overline{\mathbf{X}}=\left[\begin{array}{c}
\frac{k}{1+\alpha} \\
\frac{k}{1+\alpha}
\end{array}\right]
$$

Then with the initial conditions $\mathbf{X}^{0}(t) \equiv \overline{\mathbf{X}}$ for $t<0$, we have that $\mathbf{X}^{0}(t)=\overline{\mathbf{X}}, t>0$.

As in $\S 2.3$, the averaged noise covariance matrix is

$$
A=\left(\frac{k}{1+\alpha}\right)^{2} \sigma^{2}\left[\begin{array}{ll}
1 & \rho \\
\rho & 1
\end{array}\right] .
$$

However, because of the time lag, the previous nondelay community matrix $C$ has been decomposed into the two matrices

$$
\begin{aligned}
& C_{0}=\frac{\partial \overline{\mathbf{F}}}{\partial \mathbf{x}_{0}}(\overline{\mathbf{X}}, \overline{\mathbf{X}})=-\frac{k}{1+\alpha}\left[\begin{array}{ll}
1 & 0 \\
0 & 1
\end{array}\right], \\
& C_{1}=\frac{\partial \overline{\mathbf{F}}}{\partial \mathbf{x}_{1}}(\overline{\mathbf{X}}, \overline{\mathbf{X}})=-\frac{\alpha k}{1+\alpha}\left[\begin{array}{ll}
0 & 1 \\
1 & 0
\end{array}\right]
\end{aligned}
$$

From (3.2.3), the characteristic function is

$$
\begin{aligned}
f(s) & =\operatorname{det}\left[s I-C_{0}-e^{-\delta s} C_{1}\right] \\
& =\left(s+\frac{k}{1+\alpha}+\frac{\alpha k}{1+\alpha} e^{-\delta s}\right)\left(s+\frac{k}{1+\alpha}-\frac{\alpha k}{1+\alpha} e^{-\delta s}\right) .
\end{aligned}
$$

Let

$$
\begin{aligned}
& \lambda_{i} \text { be the } i \text { th root of } s+\frac{k}{1+\alpha}+\frac{\alpha k}{1+\alpha} e^{-s \delta}=0, \\
& \mu_{i} \text { be the } i \text { th root of } s+\frac{k}{1+\alpha}-\frac{\alpha k}{1+\alpha} e^{-s \delta}=0 .
\end{aligned}
$$

Note that if $\delta=0$ these reduce to the eigenvalues of the nondelay community matrix of $\S 2.3$. It can be shown (an application of Rouche's theorem) that if $0<\alpha<1, \delta \geqq 0$, then all these roots have negative real parts. This guarantees linear asymptotic stability of (3.3.2). For an extensive discussion of the location in the complex plane of the roots of such equations, the reader is referred to Pinney [9].

We will obtain the limiting covariance $D(\infty)$ given by (3.2.9). Accordingly, the residue matrices are, from (3.2.6),

$$
\begin{aligned}
& Y_{\lambda_{j}}=\frac{1}{2\left[1+\lambda_{j} \delta+k \delta /(1+\alpha)\right]}\left[\begin{array}{ll}
1 & 1 \\
1 & 1
\end{array}\right], \\
& Y_{\mu_{j}}=\frac{1}{2\left[1+\mu_{j} \delta+k \delta /(1+\alpha)\right]}\left[\begin{array}{rr}
1 & -1 \\
-1 & 1
\end{array}\right] .
\end{aligned}
$$


Hence

$$
\begin{aligned}
Y_{\lambda_{l}} A Y_{\lambda_{l}}^{T} & =\frac{(1+\rho) \sigma^{2}(k /(1+\alpha))^{2}}{2\left[1+\lambda_{j} \delta+k \delta /(1+\alpha)\right]\left[1+\lambda_{l} \delta+k \delta /(1+\alpha)\right]}\left[\begin{array}{ll}
1 & 1 \\
1 & 1
\end{array}\right] \\
Y_{\mu_{j}} A Y_{\mu_{l}}^{T} & =\frac{(1-\rho) \sigma^{2}(k /(1+\alpha))^{2}}{2\left[1+\mu_{j} \delta+k \delta /(1+\alpha)\right]\left[1+\mu_{l} \delta+k \delta /(1+\alpha)\right]}\left[\begin{array}{rr}
1 & -1 \\
-1 & 1
\end{array}\right], \\
Y_{\lambda_{j}} A Y_{\mu_{l}}^{T} & =Y_{\mu_{l}} A Y_{\lambda_{j}}^{T}=0 .
\end{aligned}
$$

Putting this into (3.2.9), we get

$$
D(\infty)=\frac{(1+\rho) \sigma^{2} k^{2}}{2(1+\alpha)^{2}}
$$

$$
\begin{aligned}
& \cdot \sum_{j=1}^{\infty} \sum_{l=1}^{\infty} \frac{1}{\left[1+\lambda_{j} \delta+k \delta /(1+\alpha)\right]\left[1+\lambda_{l} \delta+k \delta /(1+\alpha)\right]\left[\lambda_{j}+\lambda_{l}\right]}\left[\begin{array}{ll}
1 & 1 \\
1 & 1
\end{array}\right] \\
& +\frac{(1-\rho) \sigma^{2} k^{2}}{2(1+\alpha)^{2}} \\
& \cdot \sum_{j=1}^{\infty} \sum_{l=1}^{\infty} \frac{1}{\left[1+\mu_{j} \delta+k \delta /(1+\alpha)\right]\left[1+\mu_{l} \delta+k \delta /(1+\alpha)\right]\left[\mu_{j}+\mu_{l}\right]}\left[\begin{array}{rr}
1 & -1 \\
-1 & 1
\end{array}\right] .
\end{aligned}
$$

We rotate to the same principle coordinates used for the nondelay case, using the matrix

$$
P=\frac{1}{\sqrt{2}}\left[\begin{array}{rr}
1 & 1 \\
1 & -1
\end{array}\right]
$$

Then

$$
\hat{D}(\infty)=P^{-1} D(\infty) P=\left[\begin{array}{cc}
D_{1} & 0 \\
0 & D_{2}
\end{array}\right]
$$

where

$$
D_{1}=\frac{(1+\rho) \sigma^{2} k^{2}}{(1+\alpha)^{2}}
$$

$$
\begin{aligned}
& D_{2}=\frac{(1-\rho) \sigma^{2} k^{2}}{(1+\alpha)^{2}} \\
& \quad \cdot \sum_{j=1}^{\infty} \sum_{l=1}^{\infty} \frac{1}{\left[1+\mu_{j} \delta+k \delta /(1+\alpha)\right]\left[1+\mu_{l} \delta+k \delta /(1+\alpha)\right]\left(\mu_{j}+\mu_{l}\right)} .
\end{aligned}
$$

These expressions for $D_{1}$ and $D_{2}$ can be summed explicitly. The procedure will be sketched for $D_{1}$.

Recalling that $\lambda_{n}$ is a root of

$$
g(s)=s+\frac{k}{1+\alpha}+\frac{\alpha k}{1+\alpha} e^{-s \delta}=0
$$


we change variables, $z=(s+k /(1+\alpha)) \delta$; then $a_{n}=\left(\lambda_{n}+k /(1+\alpha)\right) \delta$ is a root of

$$
h(z)=z+\beta e^{-z}=0
$$

where

$$
\beta=\frac{\alpha k \delta}{1+\alpha} e^{k \delta /(1+\alpha)}
$$

In terms of $a_{n}$, the expression for $D_{1}$ becomes

$$
D_{1}=-\left(\frac{\sigma k}{1+\alpha}\right)^{2} \delta(1+\rho)
$$

$$
\cdot \sum_{n=1}^{\infty} \sum_{m=1}^{\infty} \frac{1}{\left(a_{n}+a_{m}-2 k \delta /(1+\alpha)\right)\left(1+a_{n}\right)\left(1+a_{m}\right)} .
$$

We make use of the identity

$$
h(z)=z+\beta e^{-z}=e^{[(1-\beta) / \beta] z} \prod_{n=1}^{\infty}\left(1-\frac{z}{a_{n}}\right) e^{z / a_{n}} .
$$

This formula is the Weierstrass infinite product representation for $h(z)$ (see Ahlfors [1]). By computing the quotient $h^{\prime}(z) / h(z)$, we obtain the identity

$$
\sum_{n=1}^{\infty}\left\{\frac{1}{z-a_{n}}+\frac{1}{a_{n}}\right\}=\frac{1-\beta e^{-z}}{z+\beta e^{-z}}+1-\frac{1}{\beta}
$$

This identity will be used repeatedly to sum the series in (3.3.13). By a partial fractions expansion of the inner sum of (3.3.13), we obtain

$$
\begin{aligned}
D_{1}=-\left(\frac{\sigma k}{1+\alpha}\right)^{2} \delta(1+\rho)\left[\sum_{n=1}^{\infty} \frac{1}{\left(1+a_{n}\right)\left(1-a_{n}+2 k \delta /(1+\alpha)\right)}\right. \\
\cdot\left\{-\sum_{m=1}^{\infty}\left(\frac{1}{\left(2 k \delta /(1+\alpha)-a_{n}-a_{m}\right)}+\frac{1}{a_{m}}\right)\right. \\
\left.\left.+\sum_{m=1}^{\infty}\left(\frac{1}{\left(-1-a_{m}\right)}-\frac{1}{a_{m}}\right)\right\}\right] .
\end{aligned}
$$

Relation (3.3.15) may now be used to sum the two series within the braces above. Simplifying, and using from the definition of $a_{n}$ that $e^{a_{n}}=-\beta / a_{n}$, we have that

$$
\begin{aligned}
D_{1}= & -\left(\frac{\sigma k}{1+\alpha}\right)^{2} \delta(1+\rho) \\
& \cdot \sum_{n=1}^{\infty} \frac{a_{n}}{\left(1+a_{n}\right)\left(a_{n}^{2}-2 k \delta /(1+\alpha) a_{n}+[\alpha k \delta /(1+\alpha)]^{2}\right)}
\end{aligned}
$$

Defining

$$
a=\frac{k \delta}{1+\alpha}\left(1+\sqrt{1-\alpha^{2}}\right), \quad b=\frac{k \delta}{1+\alpha}\left(1-\sqrt{1-\alpha^{2}}\right) .
$$


We may expand (3.3.17) in partial fractions to get

$$
\begin{aligned}
D_{1}= & \frac{\delta(1+\rho)}{(a+1)(b+1)}\left(\frac{\delta k}{1+\alpha}\right)^{2} \sum_{n=1}^{\infty}\left(\frac{1}{1+a_{n}}-\frac{1}{a_{n}}\right) \\
& -\frac{\delta(1+\rho) a}{(a+1)(a-b)}\left(\frac{\sigma k}{1+\alpha}\right)^{2} \sum_{n=1}^{\infty}\left(\frac{1}{a_{n}-a}-\frac{1}{a_{n}}\right) \\
& -\frac{\delta(1+\rho) b}{(b+1)(b-a)}\left(\frac{\delta k}{1+\alpha}\right)^{2} \sum_{n=1}^{\infty}\left(\frac{1}{a_{n}-b}-\frac{1}{a_{n}}\right) .
\end{aligned}
$$

The identity (3.3.15) may now be used again to sum each of the three series in (3.3.18). A lengthy calculation gives

$$
\begin{aligned}
D_{1}= & \frac{\sigma^{2} k(1+\rho)}{2(1+\alpha) \sqrt{1-\alpha^{2}}} \\
& \cdot \frac{\left[\sinh \left(\sqrt{1-\alpha^{2}} k \delta /(1+\alpha)\right)+\sqrt{1-\alpha^{2}} \cosh \left(\sqrt{1-\alpha^{2}} k \delta /(1+\alpha)\right)\right]}{\left[\alpha+\cosh \left(\sqrt{1-\alpha^{2}} k \delta /(1+\alpha)\right)+\sqrt{1-\alpha^{2}} \sinh \left(\sqrt{1-\alpha^{2}} k \delta /(1+\alpha)\right)\right]}
\end{aligned}
$$

A similar calculation for $D_{2}$ gives

$$
\begin{aligned}
D_{2}= & \frac{\sigma^{2} k(1-\rho)}{2(1+\alpha) \sqrt{1-\alpha^{2}}} \\
& \frac{\left[\sinh \left(\sqrt{1-\alpha^{2}} k \delta /(1+\alpha)\right)+\sqrt{1-\alpha^{2}} \cosh \left(\sqrt{1-\alpha^{2}} k \delta /(1+\alpha)\right)\right]}{\left[-\alpha+\cosh \left(\sqrt{1-\alpha^{2}} k \delta /(1+\alpha)\right)+\sqrt{1-\alpha^{2}} \sinh \left(\sqrt{1-\alpha^{2}} k \delta /(1+\alpha)\right)\right]} .
\end{aligned}
$$

For $\delta=0$, these expressions reduce, as expected, to the nondelay results of $\S 2.3$. The effect of the time delay on the variance of the fluctuations is, in fact, surprisingly undramatic. By differentiation, we obtain

$$
\frac{\partial D_{1}}{\partial \delta}=\frac{(\alpha(1+\rho) / 2)(\sigma k /(1+\alpha))^{2}}{\left.\left.\left[\alpha+\cosh \left(\sqrt{1-\alpha^{2}} k \delta\right) /(1+\alpha)\right)+\sqrt{1-\alpha^{2}} \sinh \left(\left(\sqrt{1-\alpha^{2}}\right) /(1+\alpha)\right) k \delta\right)\right]},
$$

$$
\frac{\partial D_{2}}{\partial \delta}=\frac{(-\alpha(1-\rho) / 2)(\sigma k /(1+\alpha))^{2}}{\left[-\alpha+\cosh \left(\left(\sqrt{1-\alpha^{2}} k \delta\right) /(1+\alpha)\right)+\sqrt{1-\alpha^{2}} \sinh \left(\left(\sqrt{1-\alpha^{2}}\right) /(1+\alpha) k \delta\right)\right]} .
$$

Thus, $\partial D_{1} / \partial \delta>0$, and $D_{1}$ increases with $\delta$ to a maximum value:

$$
D_{1} \uparrow \frac{\sigma^{2} k(1+\rho)}{2(1+\alpha) \sqrt{1-\alpha^{2}}} \text { as } \delta \text { increases. }
$$

Similarly $\partial D_{2} / \partial \delta<0$, and

$$
D_{2} \downarrow \frac{\sigma^{2} k(1-\rho)}{2(1+\alpha) \sqrt{1-\alpha^{2}}} \text { as } \delta \text { increases. }
$$


Thus, while the delay increases the component $D_{1}$, the variance of the sum of the populations, it also decreases the component $D_{2}$, the variance of the difference of the populations. In the case $\rho=0$ (species environments uncorrelated), we have $D_{1}<D_{2}$ for all parameter values, with larger delays tending to bring $D_{1}$ and $D_{2}$ closer to equality. Therefore, if ellipses are drawn about the equilibrium point to represent the contour lines of the Gaussian probability cloud, then the effect of the delay is merely to decrease the eccentricity of the ellipse.

3.4. The delay sensitivity matrix. Consider a system with a stable equilibrium point, and a small time lag effect, which we are tempted to ignore in writing the equations. If the delay is sufficiently small, it will not, in general destroy the stability in a deterministic environment (see Halany [4]). However, it may be necessary to assess the effects of the delay on the fluctuations of the populations in a random environment.

Let $D(t ; \delta)$ be the covariance of the fluctuations $Y^{0}(t)$ for a model with delay $\delta$. If $\delta$ is small, we may make the Taylor series approximation

$$
D(t ; \delta)=D(t ; 0)+\frac{\partial D}{\partial \delta}(t ; 0) \delta+(\text { higher order in } \delta) .
$$

Thus, a first order correction can be made to the nondelay model by calculating $(\partial D / \partial \delta)(t ; 0)$. The ultimate stochastic effects may be checked by looking at this correction for the equilibrium variance $D(\infty ; 0)$. Therefore we define the sensitivity matrix as

$$
S(t)=\frac{\partial D}{\partial \delta}(t ; 0)
$$

By differentiating (3.1.10) and (3.2.2) with respect to $\delta$, it can be shown that

$$
S(t)=S_{0}(t)+S_{0}^{T}(t)
$$

where

$$
S_{0}(t)=-\left\{C_{1} D(t ; 0)+\int_{0}^{t} e^{C s} C_{1} C e^{-C s}[D(t ; 0)-D(s ; 0)] d s\right\}
$$

Here $C$ is the community matrix for the nondelay model, and $C_{1}$ is the matrix of derivatives with respect to delay variables calculated from (3.1.5).

In the special case when $C$ and $C_{1}$ commute, the expression reduces to

$$
\left.S_{0}(t)=-C_{1}\left\{D(t ; 0)+C \int_{0}^{t} D(t ; 0)-D(s ; 0)\right] d s\right\}
$$

This is the case for the competing species model of $\S 3.3$, which was analyzed without delays in $\S 2.3$. Insertion of equations (2.3.9), (2.3.10) and (3.3.5) into (3.4.3') yields for $t=\infty$ (and in principal coordinates),

$$
\hat{S}(\infty)=\left[\begin{array}{cc}
\frac{\alpha(1+\rho)}{2(1+\alpha)}\left(\frac{\sigma k}{1+\alpha}\right)^{2} & 0 \\
0 & \frac{-\alpha(1-\rho)}{2(1-\alpha)}\left(\frac{\sigma k}{1+\alpha}\right)^{2}
\end{array}\right] .
$$


This agrees with the direct computations (3.3.21). Thus, without the more difficult computations of $\S 3.3$, we can see that the delay increases the first component and decreases the second, and that if $\alpha$ is close to one, the decrease in the second component is substantial.

3.5. Systems with random delay. All time-delays considered previously were fixed and nonrandom. We will indicate here how the calculations change if the time lag is itself a rapidly-varying random process. More complete results are in [12].

For simplicity, we will consider the case when the delay process $\delta(\tau), \tau=t / \varepsilon$ is the only random term.

$$
\frac{d}{d t} \mathbf{X}^{\varepsilon}(t)=\mathbf{F}\left(\mathbf{X}^{\varepsilon}(t-\delta(t / \varepsilon))\right) .
$$

We will further let $\delta(\tau)$ be a stationary process, with distribution function $\bar{P}(s)=P\{\delta(\tau)<s\}$, and assume it is bounded by some constant $a, 0 \leqq \delta(\tau) \leqq a$ with probability one. Then some initial conditions must be specified for $\mathbf{X}^{\varepsilon}(t)$ for $t \in[-a, 0]$.

The deterministic trajectory $\mathbf{X}^{0}(t)$ satisfies

$$
\frac{d}{d t} \mathbf{X}^{0}(t)=\int_{0}^{a} \mathbf{F}\left(\mathbf{X}^{0}(t-s)\right) d \bar{P}(s) .
$$

with the same initial conditions as $\mathbf{X}^{\varepsilon}(t)$. Thus a nonlinear integro-differential equation replaces the random delay-differential equation to first approximation.

Define the correlation matrix $A(t)$ by

$$
\begin{array}{r}
=\lim _{T \rightarrow \infty} \frac{1}{T} \int_{0}^{T} d s_{1} \int_{0}^{T} d s_{2} E\left\{\left[F\left(X^{0}\left(t-\delta\left(\tau_{1}\right)\right)\right)-E\left\{F\left(X^{0}\left(t-\delta\left(\tau_{1}\right)\right)\right)\right\}\right]\right. \\
\left.\cdot\left[F\left(X^{0}\left(t-\delta\left(\tau_{2}\right)\right)\right)-E\left\{F\left(X^{0}\left(t-\delta\left(\tau_{2}\right)\right)\right)\right\}\right]^{T}\right\}
\end{array}
$$

Let $W(t)$ be a white noise with correlation matrix $A(t) \delta\left(t-t^{\prime}\right)$.

Now suppose the distribution function $\bar{P}(s)$ gives nonzero masses $\mu_{i}$ to the discrete set of points $\delta_{i}, i=1,2, \cdots, N$, and has a continuous part $P_{0}(s)$. Then we may approximate

$$
\mathbf{X}^{\varepsilon}(t)=\mathbf{X}^{0}(t)+\sqrt{\varepsilon} \mathbf{Y}^{0}(t),
$$

where the Gaussian random process $\mathbf{Y}^{0}(t)$ satisfies the linear white noise equation

$$
\begin{aligned}
Y^{0}(t)= & \int_{0}^{t} W(s) d s+\sum_{i=1}^{N} \mu_{i} \int_{0}^{t-\delta_{i}} \frac{\partial \mathbf{F}}{\partial x}\left(X^{0}(s)\right) Y^{o}(s) d s \\
& +\int_{0}^{t} \bar{P}_{0}(t-s) \frac{\partial \mathbf{F}}{\partial x}\left(X^{0}(s)\right) Y^{0}(s) d s .
\end{aligned}
$$

If $\delta(\tau)$ takes only the discrete values $\delta_{i}$ we get a delay-differential equation by setting $\bar{P}_{0} \equiv 0$ and differentiating (3.5.5). Otherwise, $\mathbf{Y}^{0}(t)$ satisfies a Volterra 
integral equation with stochastic inhomogeneous term $\int_{0}^{t} W(s) d s$. The solution can be written in terms of the deterministic reciprocal kernel of (3.5.5), and formulas derived analogous to those of the previous sections.

As an example we consider a delay process $\delta(\tau)$ which is a finite state ergodic Markov chain taking values in the set $\left\{0 \equiv \delta_{0}<\delta_{1}<\cdots<\delta_{N}\right\}$. We assume stationary transition probabilities $P_{i j}(\tau)$ defined by

$$
P_{i j}\left(\tau_{2}-\tau_{1}\right)=P_{r}\left\{\delta\left(\tau_{2}\right)=\delta_{j} \mid \delta\left(\tau_{1}\right)=\delta_{i}\right\} \quad \text { for } \tau_{1}<\tau_{2} .
$$

We furthermore assume the process has reached its equilibrium distribution $\Pi_{i}$, i.e.

$$
\Pi_{i}=P_{r}\left\{\delta(\tau)=\delta_{i}\right\} \text { for all } \tau .
$$

Then the first approximation $\mathbf{X}^{0}(t)$ satisfies

$$
\frac{d}{d t} \mathbf{X}^{0}(t)=\sum_{i=0}^{N} \Pi_{i} \mathbf{F}\left(\mathbf{X}^{0}\left(t-\delta_{i}\right)\right)
$$

The fluctuations $\mathbf{Y}^{0}(t)$ satisfy

$$
\frac{d}{d t} Y^{0}(t)=W(t)+\sum_{i=0}^{N} C_{i}(t) Y^{0}\left(t-\delta_{i}\right)
$$

where

$$
C_{i}(t)=\Pi_{i} \frac{\partial \mathbf{F}}{\partial \mathbf{x}}\left(\mathbf{X}^{0}\left(t-\delta_{i}\right)\right)
$$

Here $W(t)$ is a Gaussian white noise with covariance $A(t) \delta\left(t-t^{\prime}\right)$, and $A(t)$ is defined by

$$
\begin{aligned}
A(t)=\sum_{i=0}^{N} \sum_{j=0}^{N} \mathbf{F}\left(\mathbf{X}^{0}\left(t-\delta_{i}\right)\right) \mathbf{F}^{T}\left(\mathbf{X}^{0}\left(t-\delta_{j}\right)\right) \\
\cdot\left\{\Pi_{i} \int_{0}^{\infty}\left[P_{i j}(s)-\Pi_{j}\right] d s+\Pi_{j} \int_{0}^{\infty}\left[P_{j i}(s)-\Pi_{i}\right] d s\right\}
\end{aligned}
$$

4.1. Derivation of the method. In this section we give a heuristic derivation of the method. Rigorous proofs are in [5], [12]. For simplicity, we discuss the random equation

$$
\begin{aligned}
\frac{d}{d t} \mathbf{X}^{\varepsilon}(t) & =\mathbf{F}\left(\mathbf{X}^{\varepsilon}(t) ; \boldsymbol{\gamma}(t / \varepsilon)\right), \\
\mathbf{X}^{\varepsilon}(0) & =\mathbf{x}_{0}
\end{aligned}
$$

where $\gamma(\tau)$ is a stochastic process, rapidly-fluctuating on the fast time scale $\tau=t / \varepsilon$.

For fixed $\mathbf{x}$, consider the random process $\mathbf{F}(\mathbf{x} ; \boldsymbol{\gamma}(\tau))$. With some technical hypotheses, this process will satisfy a law of large numbers, i.e., there is some 
deterministic function $\overline{\mathbf{F}}(\mathbf{x})$ such that for every $t_{0}$,

$$
\frac{1}{T} \int_{t_{0}}^{t_{0}+T} \mathbf{F}(\mathbf{x} ; \boldsymbol{\gamma}(\tau)) \rightarrow \overline{\mathbf{F}}(\mathbf{x}) \quad \text { as } T \rightarrow \infty .
$$

The convergence in (4.1.2) is in some probabilistic sense. We interpret it here as holding in the mean, i.e.,

$$
\lim _{T \rightarrow \infty} E\left\{\frac{1}{T}\left|\int_{t_{0}}^{t_{0}+T} \mathbf{F}(\mathbf{x} ; \boldsymbol{\gamma}(\tau)) d \tau-\mathbf{F}(\mathbf{x})\right|\right\}=0 .
$$

As an example of this, consider the special case of a one-dimensional $F$ of the form

$$
F(x ; \gamma(\tau))=F_{0}(x)+F_{1}(x) \gamma(\tau)
$$

Here $F_{0}$ and $F_{1}$ are deterministic, $\gamma(\tau)$ is a mean zero, wide sense stationary process with covariance

$$
R(u)=E[\gamma(\tau) \gamma(\tau+u)]
$$

Then setting $\bar{F} \equiv F_{0}$,

$$
\begin{array}{rl}
E \mid \frac{1}{T} \int_{t_{0}}^{t_{0}+T} & F(x ; \gamma(\tau)) d \tau-\bar{F}(x) \mid \\
& =\left|F_{1}(x)\right| E\left|\frac{1}{T} \int_{t_{0}}^{t_{0}+T} \gamma(\tau) d \tau\right| \\
& \leqq\left|F_{1}(x)\right|\left[\frac{1}{T^{2}} \int_{t_{0}}^{t_{0}+T} \int_{t_{0}}^{t_{0}+T} E\left[\gamma\left(\tau_{1}\right) \gamma\left(\tau_{2}\right)\right] d \tau_{1} d \tau_{2}\right]^{1 / 2} \\
& =\left|F_{1}(x)\right|\left[\frac{2}{T} \int_{0}^{T}\left(1-\frac{u}{T}\right) R(u) d u\right]^{1 / 2} .
\end{array}
$$

This expression converges to zero if, say

$$
\int_{0}^{\infty} R(u) d u<\infty
$$

Condition (4.1.5) is an assertion on how quickly correlations between $\gamma(\tau)$ and $\gamma(\tau+u)$ die out as the time separation $u$ increases to infinity. In the case of general $\mathbf{F}$ this condition must be strengthened to a "mixing" hypothesis, that events separated in time are asymptotically independent. Roughly, the randomness must forget itself at a sufficiently rapid rate.

Returning to the solution of (4.1.1), assume that (4.1.2') holds for some function $\overline{\mathbf{F}}(\mathbf{x})$. Then clearly $\overline{\mathbf{F}}$ must satisfy

$$
\overline{\mathbf{F}}(x)=\lim _{T \rightarrow \infty} \frac{1}{T} \int_{t_{0}}^{t_{0}+T} E \mathbf{F}(x ; \gamma(\tau)) d \tau .
$$


In integrated form, (4.1.1) becomes

$$
\mathbf{X}^{\varepsilon}(t)=\mathbf{x}_{0}+\int_{0}^{t} \mathbf{F}\left(\mathbf{x}^{\varepsilon}(s) ; \gamma(s / \varepsilon)\right) d s .
$$

The integral on the right depends both on the statistics of $F(\mathbf{x} ; \boldsymbol{\gamma}(s / \varepsilon))$, and those of the unknown solution $\mathbf{X}^{\varepsilon}(s)$. We can, however, analyze the integral if $\mathbf{X}^{\varepsilon}(s)$ is replaced by some continuous deterministic function $\mathrm{z}(s)$. Let $n$ be a large integer, $\Delta=t / n, t_{i}=i \Delta$, for $i=0,1, \cdots, n$. Then if $n$ is large enough so that $\mathrm{z}$ changes little on an interval of length $\Delta$,

$$
\begin{aligned}
\int_{0}^{t} \mathbf{F}(\mathbf{z}(s) ; \boldsymbol{\gamma}(s / \varepsilon)) d s & =\sum_{i=0}^{n} \int_{t_{i}}^{t_{i}+\Delta} \mathbf{F}(\mathbf{z}(s) ; \gamma(s / \varepsilon)) d s \\
& \approx \sum_{i=0}^{n=1} \int_{t_{i}}^{t_{i}+\Delta} \mathbf{F}\left(\mathbf{z}\left(t_{i}\right) ; \gamma(s / \varepsilon)\right) d s \\
& =\sum_{i=0}^{n=1} \varepsilon \int_{t_{i} / \varepsilon}^{t_{i} / \varepsilon+\Delta / \varepsilon} F\left(z\left(t_{i}\right) ; \gamma(\tau)\right) d \tau \\
& =\sum_{i=0}^{n-1} \Delta \frac{1}{T} \int_{t_{i} / \varepsilon}^{t_{i} / \varepsilon+T / \varepsilon} \mathbf{F}\left(\mathbf{z}\left(t_{i}\right) ; \gamma(\tau)\right) d \tau .
\end{aligned}
$$

Here $T=\Delta / \varepsilon$ is large for $\varepsilon$ small; so using (4.1.2),

$$
\int_{0}^{t} \mathbf{F}(\mathbf{z}(s) ; \boldsymbol{\gamma}(s / \varepsilon)) d s \approx \sum_{i=0}^{n-1} \Delta \overline{\mathbf{F}}\left(z\left(t_{i}\right)\right)
$$

This last expression is a Riemann approximation to an integral, so

$$
\int_{0}^{t} \mathbf{F}(\mathbf{z}(s) ; \boldsymbol{\gamma}(s / \varepsilon)) d s \rightarrow \int_{0}^{t} F(\mathbf{z}(s)) d s .
$$

Now define $\mathbf{X}^{0}(t)$ as the solution of

$$
\begin{aligned}
\frac{d}{d t} \mathbf{X}^{0}(t) & =\overline{\mathbf{F}}\left(X^{o}(t)\right), \\
\mathbf{X}^{0}(0) & =x_{0} .
\end{aligned}
$$

Then substituting $\mathbf{X}^{0}$ for $\mathbf{X}^{\varepsilon}$ on the right of (4.1.7), we obtain

$$
x_{0}+\int_{0}^{t} \mathbf{F}\left(\mathbf{X}^{0}(s) ; \gamma(s / \varepsilon)\right) d s \approx x_{0}+\int_{0}^{t} \overline{\mathbf{F}}\left(\mathbf{X}^{o}(s)\right) d s=\mathbf{X}^{0}(t) .
$$

so $\mathbf{X}^{0}$ is an approximate solution of (4.1.7). One can in fact show that

$$
E\left|X^{\varepsilon}(t)-X^{0}(t)\right| \rightarrow 0 \quad \text { as } \varepsilon \rightarrow 0 .
$$

In the degenerate case when $\gamma(\tau)$ is a nonrandom function, the expectation in (4.1.6) is superfluous, and the approximation $\mathbf{X}^{0}(t)$ reduces to the well-known deterministic "method of averaging".

To get a first order stochastic correction to $\mathbf{X}^{0}(t)$, we expand

$$
\mathbf{X}^{\varepsilon}(t)=\mathbf{X}^{0}(t)+\varepsilon^{\alpha} \mathbf{Y}^{0}(t)+(\text { higher order in } \varepsilon) .
$$


The leading order, $\alpha>0$, of the expansion is to be determined. Expanding

$$
\begin{aligned}
\mathbf{F}\left(X^{\varepsilon}(t) ; \gamma(\tau)\right) & =\mathbf{F}\left(X^{0}+\varepsilon^{\alpha} Y^{0}+\cdots ; \gamma(\tau)\right) \\
& =\mathbf{F}\left(X^{0} ; \gamma\right)+\varepsilon^{\alpha} \frac{\partial \mathbf{F}}{\partial \mathbf{x}}\left(X^{0} ; \gamma\right) Y^{0}+\cdots,
\end{aligned}
$$

we get from (4.1.1),

$$
\begin{aligned}
& \frac{d}{d t} \mathbf{X}^{0}(t)+\varepsilon^{\alpha} \frac{d}{d t} \mathbf{Y}^{0}(t)+\cdots \\
& \mathbf{F}\left(\mathbf{X}^{0}(t) ; \gamma(t / \varepsilon)\right)+\varepsilon^{\alpha} \frac{\partial \mathbf{F}}{\partial \mathbf{x}}\left(X^{0}(t) ; \boldsymbol{\gamma}(t / \varepsilon)\right) Y^{0}(t)+\cdots
\end{aligned}
$$

Note that equating powers of $\varepsilon$ in (4.1.12) does not give the correct result in (4.1.9). Of course epsilons still occur within the functions $F$ and $\partial \mathbf{F} / \partial x$. Assuming that higher order terms may be neglected, we substitute for $(d / d t) \mathbf{X}^{0}(t)$ from (4.1.9). Then integration of the equation yields

$$
\begin{aligned}
\mathbf{Y}^{0}(t)= & \frac{1}{\varepsilon^{\alpha}} \int_{0}^{t}\left[F\left(\mathbf{X}^{0}(s) ; \gamma(s / \varepsilon)\right)-\bar{F}\left(\mathbf{X}^{0}(d)\right)\right] d s \\
& +\int_{0}^{t} \frac{\partial \mathbf{F}}{\partial x}\left(\mathbf{X}^{0}(s) ; \gamma(s / \varepsilon)\right) \mathbf{Y}^{0}(s) d s .
\end{aligned}
$$

A central limit theorem for mixing processes now dictates that $\alpha=\frac{1}{2}$; i.e., if

$$
\mathbf{G}^{\varepsilon}(t)=\frac{1}{\sqrt{\varepsilon}} \int_{0}^{t}\left[\mathbf{F}\left(\mathbf{X}^{0}(s) ; \boldsymbol{\gamma}(s / \varepsilon)\right)-\overline{\mathbf{F}}\left(\mathbf{X}^{0}(s)\right)\right] d s,
$$

then $\mathbf{G}^{\varepsilon}(t)$ converges (weakly) as $\varepsilon \rightarrow 0$ to a Gaussian process, with mean zero, and a covariance matrix that can be calculated by taking the limit of $E\left\{G_{i}^{\varepsilon}(t) G_{j}^{\varepsilon}(t)\right\}$. We denote the limit process by $\mathbf{G}^{0}(t)$.

Now

$$
\frac{\partial \mathbf{F}}{\partial \mathbf{X}}\left(\mathbf{X}^{0}(s) ; \gamma(s / \varepsilon)\right) \rightarrow \frac{\partial \overline{\mathbf{F}}}{\partial \overline{\mathbf{X}}}\left(\mathbf{X}^{0}(s)\right)
$$

by the same argument as given for the convergence of $\mathbf{F}\left(\mathbf{X}^{0}(s) ; \boldsymbol{\gamma}(s / \varepsilon)\right) \rightarrow \overline{\mathbf{F}}\left(\mathbf{X}^{0}(s)\right)$. Therefore, we have the limiting equation

$$
\mathbf{Y}^{0}(t)=\mathbf{G}^{0}(t)+\int_{0}^{t} \frac{\partial \overline{\mathbf{F}}}{\partial X}\left(\mathbf{X}^{0}(s)\right) \mathbf{Y}^{0}(s) d s .
$$

Since the derivative of $G^{0}(t)$ is a white noise $W(t)$ we have in differential form

$$
\begin{gathered}
\frac{d}{d t} \mathbf{Y}^{0}(t)=\mathbf{W}(t)+\frac{\partial \overline{\mathbf{F}}}{\partial \mathbf{X}}\left(\mathbf{X}^{0}(s)\right) \mathbf{Y}^{0}(s), \\
\mathbf{Y}^{\mathbf{0}}(0)=0 .
\end{gathered}
$$


Acknowledgments. It is a pleasure to thank Professors George Papanicolaou and Donald Ludwig for many helpful conversations, and Professors Papanicolaou and J. B. Keller for their comments on the manuscript.

\section{REFERENCES}

[1] L. V. Ahlfors, Complex Analysis, McGraw-Hill, New York, 1953.

[2] E. A. CoDdington AND N. Levinson, Theory of Ordinary Differential Equations, McGrawHill, New York, 1955.

[3] I. I. GIKHMAN AND A. V. SKorokHOD, Stochastic Differential Equations, Springer-Verlag, New York, 1972.

[4] A. Halany, Differential Equations, Academic Press, New York, 1966.

[5] R. Z. KHASMINSKII, Stochastic processes defined by differential equations, Theory Prob. Appl. 9 (1966), pp. 211-228.

[6] D. LuDwIG, Persistence of dynamical systems under random perturbations, SIAM Rev., 17 (1975), pp. 605-639.

[7] R. M. MAY, Stability and Complexity in Model Ecosystems, Princeton University Press, Princeton, NJ, 1973.

[8] - Stability in randomly fluctuating versus deterministic environments, American Naturalist, 107, (1973), no. 957.

[9] E. Pinney, Ordinary Difference-Differential Equations, Univ. of California Press, Berkeley, 1958.

[10] J. H. VANDERMEER, The community matrix and the number of species in a community, American Naturalist, 104 (1970), pp. 73-83.

[11] A. D. VENTZEL AND M. I. FREIDLIN, On small random perturbations of dynamical systems, Uspehi Mat. Nauk., 25 (1970), no. 1.

[12] B. S. WhIte, Some limit theorems for stochastic delay-differential equations, Comm. Pure Appl. Math., 29 (1976), pp. 113-141.

[13] - Some limit theorems for stochastic delay-differential equations, with applications to theoretical ecology, Ph.D. thesis, New York Univ., New York, October, 1974. 